\title{
AVALIAÇÃO COMPUTACIONAL DA INFLUÊNCIA DA TEMPERATURA NA POTÊNCIA DE UM PAINEL FOTOVOLTAICO
}

\author{
Indira Ponte Ribeiro, Francisco Frederico dos SAntos Matos, \\ AUZUIR RIPARDO ALEXANDRIA \\ Instituto Federal de Educação, Ciência e Tecnologia do Ceará (IFCE) \\ Programa de Pós-graduação em Energias Renováveis (PPGER) \\ <indriraponteribeiro@gmail.com>.<ffsmatos@ifce.edu.br>.<auzuir@ifce.edu.br>
}

DOI: 10.21439/conexoes.v10i5.1160

\begin{abstract}
Resumo. Este trabalho apresenta a análise da influência da temperatura na eficiência de um painel solar, sob condições de irradiação constante. As geometrias usadas na simulação foram criadas no software ICEM, usando dimensões reais de um painel solar, que foram então processadas no software ANSYS CFX. O objetivo deste trabalho é avaliar o percentual de perda de potência causado pelo aumento da temperatura. Simulou-se a temperatura na interface do painel em dois casos distintos: com e sem a presença de circulação de ar. Os resultados mostram uma redução gradual do percentual de perda de potência, em função da diminuição da temperatura do painel, conforme se resfria a interface entre os domínios. Ao se comparar o percentual de perda de potência das simulações que apresentaram a maior e a menor temperatura no painel, obteve-se uma redução de $75 \%$ da queda de potência.
\end{abstract}

Palavras-chaves: Desempenho de painéis fotovoltaicos. Simulação computacional. Energia solar.

\begin{abstract}
This work presents the influence of temperature on the efficiency of a solar panel under conditions of constant irradiation. The geometries used in simulation were created in the software ICEM, using the real dimensions of a photovoltaic panel, which were in turn processed in the software ANSYS CFX. The objective of this study is to evaluate the power loss percentage caused by the increase in temperature. The temperature was simulated in the panel interface for two different cases: with and without the presence of air circulation. The results show a gradual reduction of power loss percentage, due to the decrease the temperature of the panel, as the boundary interface cools down. When comparing the power loss percentage of the simulations that showed the highest and the lowest temperature in the panel, it was obtained a $75 \%$ reduction of power loss.
\end{abstract}

Keywords: Performance of photovoltaic panel. Computer simulation. Solar energy.

\section{INTRODUÇÃO}

O Sol é a maior fonte de energia da Terra, responsável por fornecer $1,5 \cdot 10^{18} \mathrm{kWh}$ de energia anualmente, o que corresponde a $10^{4}$ vezes o total de energia produzida neste mesmo período (CRESESB/CEPEL, 2006).

Um dos diversos métodos de aproveitamento da energia solar é conseguido através do uso de painéis fotovoltaicos, cujas características elétricas são determinadas nas condições padrões de teste, ou seja, com temperatura na célula de $25{ }^{\circ} \mathrm{C}$, distribuição espectral de 1,5 AM e irradiância de $1000 \mathrm{~W} / \mathrm{m}^{2}$. Esta padronização permite uma comparação de módulos de diversos fabricantes de acordo com os mesmos critérios. No entanto, estas condições dificilmente serão obtidas fora dos laboratórios de ensaio. Para a temperatura ambiente de $20^{\circ} \mathrm{C}$, por exemplo, a célula fotovoltaica atinge, em média, $48,4{ }^{\circ} \mathrm{C}$ (VILLALVA; GAZOLI, 2012). Além da temperatura na célula, outras variáveis como ângulo de incidência, influência da radiação difusa e distribuição espectral diferente de 1,5 AM não são levadas em consideração ao se calcular os parâmetros de desempenho de um painel. Desta forma, os painéis sempre apresentarão um desempenho inferior ao especificado nas folhas de dados (SACRAMENTO et al., 2015, CARDONA; LÓPEZ, 1999).

Em estudo de avaliação do desempenho de painéis fotovoltaicos expostos a condições reais, em um pe- 
ríodo de teste de 17 meses, Gxasheka, Dyk e Meyer (2005) concluiu que células trabalhando em temperaturas próximas de $40^{\circ} \mathrm{C}$ apresentam redução de até $7 \%$ na potência de saída.

Gnoatto et al. (2005) desenvolveu um estudo para determinar a curva característica de painéis sob condições reais de campo, obtendo como resultados perdas de $24 \%$ em seu rendimento em relação do padrão STC. MICHELS et al.(2008) estudou o efeito da temperatura na potência de saída de painéis fotovoltaicos de silício policristalino, e observou perdas de potência aproximadamente $6 \%$ em painéis com temperatura $15^{\circ} \mathrm{C}$ acima das condições padrões de teste.

Este artigo visa a analisar da influência da temperatura na eficiência de um painel fotovoltaico, utilizando simulações feitas no software ANSYS CFX. O presente trabalho é dividido em seções: na seção 1 é apresentada a revisão bibliográfica do tema, na seção 2 discorre-se sobre a metodologia do estudo, em seguida, na seção 3 são apresentados e comentados os resultados do estudo e, por fim, na seção 4 as conclusões são extraídas.

\section{FUNDAMENTAÇÃO TEÓRICA}

\subsection{Curvas Características De Painéis Fotovoltai- $\cos$}

Um painel fotovoltaico não se comporta como uma fonte de corrente convencional. O ponto de operação do módulo fotovoltaico, ou seja, os valores de tensão e corrente nos seus terminais, depende do que está conectado aos seus terminais. A relação entre tensão e corrente na saída é representada através das suas curvas características, nas quais são apresentados os pontos de máxima potência, ponto de corrente de curto-circuito e ponto de tensão de circuito aberto. Para cada curva I-V existe uma curva potência-tensão correspondente. Essas curvas características são obtidas nas condições padrões de teste (STC), ou seja, com irradiância solar de $1000 \mathrm{~W} / \mathrm{m}^{2}$, temperatura de $25^{\circ} \mathrm{C}$ na célula e distribuição espectral de 1,5 AM (GUENOUNOU; MALEK; AILLERIE, 2016).

Estão presentes implicitamente nas curvas características radiação e temperatura, os fatores que influenciam fortemente no desempenho dos painéis fotovoltaicos (GOKILAVANI; PRABHAKARAN; KANNADASAN, 2014).

A dependência da curva I-V destas variáveis climáticas é apresentada na Figura 1 Considerando-se a radiação constante, percebe-se que aumentos na temperatura há um pequeno aumento da corrente de curtocircuito, ocasionada pelo aumento do número de portadores de carga liberados pelo estímulo térmico. No entanto, esse aumento na corrente não compensa a queda nos valores da tensão de circuito aberto. Logo, ocorre redução na potência total. A queda de potência máxima é de aproximadamente $-0,5 \% /{ }^{\circ} \mathrm{C}$ (BORGES NETO; CARVALHO, 2012).

\section{METODOLOGIA}

Este trabalho se baseia em simulações de um painel fotovoltaico exposto ao ar a $27^{\circ} \mathrm{C}$, posicionado paralelo ao plano horizontal, em dois casos distintos, a saber, com e sem ventilação, a fim de comparar as temperaturas na superfície do painel em todos os casos. Para o estudo da transferência de calor, gerado por fluxo radiante, do ar para o painel, fez-se necessário criar duas geometrias distintas, sendo uma para o fluido e outra para o sólido. Ambas as geometrias foram criadas usando o software ICEM e simuladas no ANSYS CFX.

O painel usado como base para a modelagem foi um de $260 \mathrm{~W}$, do fabricante Yingli, cujas dimensões são $165 \times 5 \times 99 \mathrm{~cm}$. O bloco correspondente a camada de ar foi desenhado para que se situasse imediatamente acima da geometria do painel. Foi adotada uma camada de ar com espessura de $20 \mathrm{~cm}$, dando ao bloco as dimensões 165x20x99 cm. Na Figura 2 é apresentado o modelo utilizado nas simulações.

Para se configurar a simulação, é necessário especificar as condições iniciais, as condições de contorno e determinar o modelo do fluxo de radiação.Uma vez que o estudo envolve blocos de naturezas físicas distintas, criaram-se dois domínios.

O material escolhido para compor a estrutura do domínio do painel foi o alumínio. Todas as fronteiras deste domínio, excetuando-se a que está em contato com o domínio do ar foram configuradas como wall, fronteiras sólidas para o fluxo do fluido, mas que permitem a passagem de calor e de outras variáveis para dentro e para fora do domínio. Consideraram-se estas fronteiras adiabáticas.

O domínio do ar foi inicializado com temperatura de $27^{\circ} \mathrm{C}$. A radiação aparece nesse domínio como termo fonte, com fluxo no valor de $200 \mathrm{~W} / \mathrm{m}^{2}$, considerando uma incidência normal à placa. Nas simulações em que não existe fluxo de ar com velocidade diferente de zero, todas as fronteiras, exceto a que está em contato com o painel, foram configuradas como opening, ou seja, fronteiras que permitem que o fluido atravesse a superfície em qualquer direção, com pressão relativa de $0 \mathrm{~Pa}$. Quando há circulação de ar, uma das fronteiras inicialmente configuradas como opening se torna inlet, ou seja, fronteira na qual o fluido é predominantemente dirigido para o domínio, com fluido a temperatura de $25^{\circ} \mathrm{C}$ e velocidade variando de $2,5 \mathrm{~m} / \mathrm{s}$ a 7,5 $\mathrm{m} / \mathrm{s}$, propagando-se na direção do eixo $X$. 

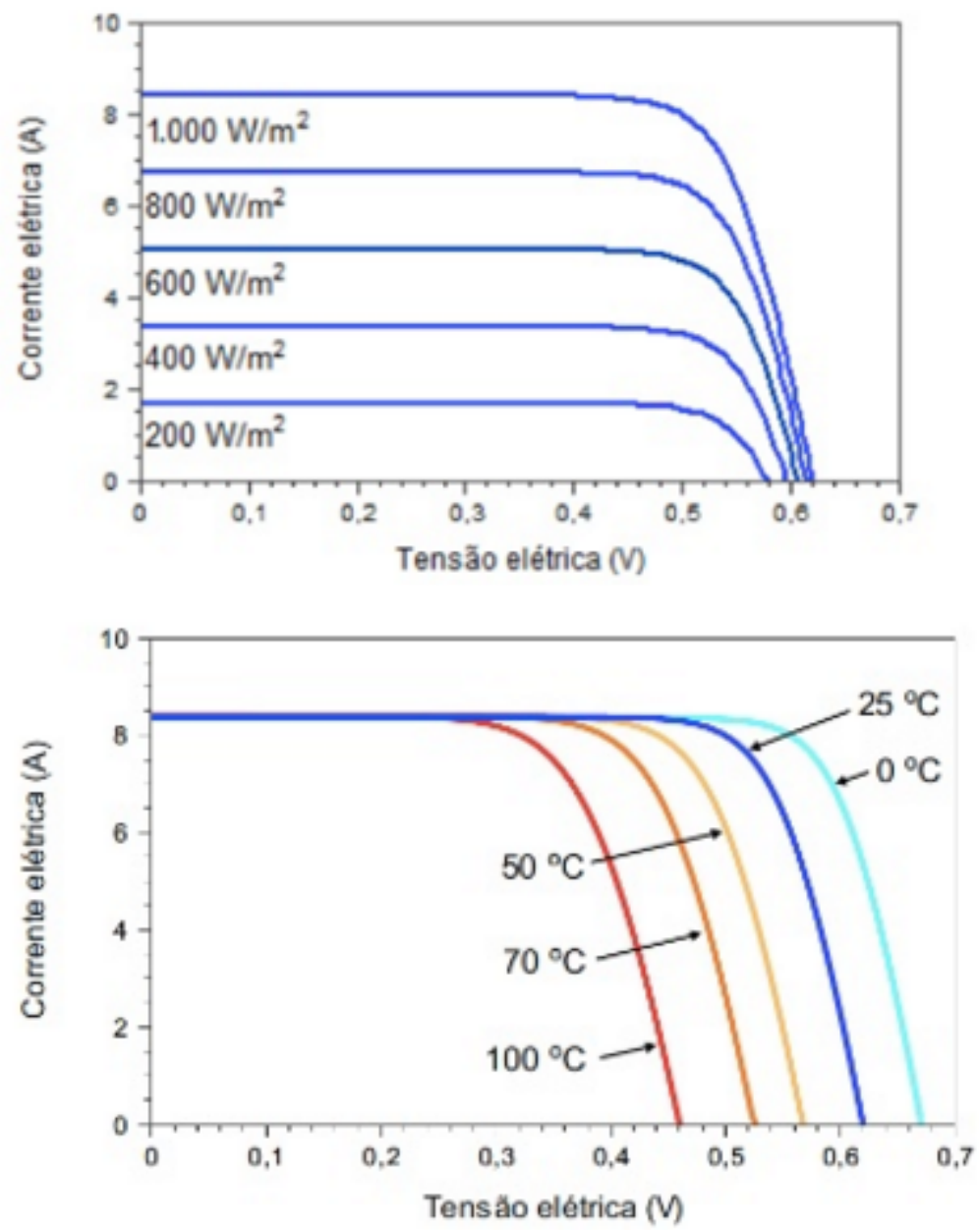

Figura 1: Curvas características de um painel solar. Fonte:Manual de Engenharia para Sistemas Fotovoltaicos (GTES, 2014).

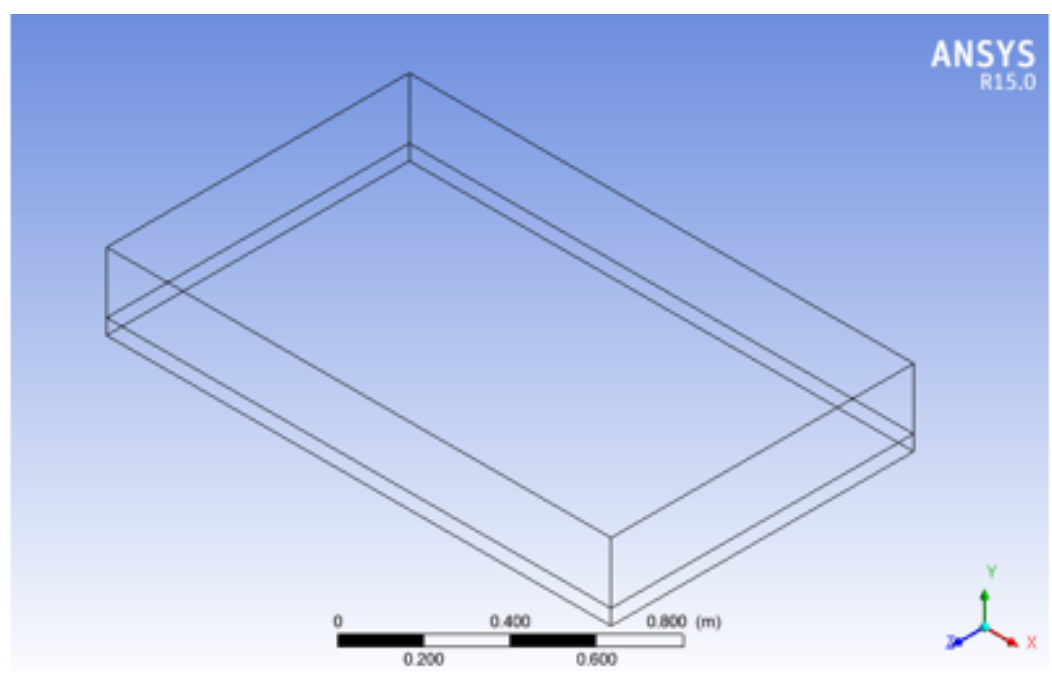

Figura 2: Modelo da simulação com domínio do ar(acima) e do painel(abaixo).

Conex. Ci. e Tecnol. Fortaleza/CE, v. 10, n. 5, p. 28 - 33, dez. 2016 
Para a modelagem da radiação, apenas o modelo Monte Carlo é aplicável, pois este é o modelo habilitado para simulações de radiação em sólidos. Como ambos os domínios devem possuir o mesmo modelo de radiação, este foi o modelo escolhido. Ainda na modelagem da radiação, é necessário definir o modelo espectral. Conforme recomendações dos tutoriais, o modelo escolhido foi o gray, por assumir uniformidade na quantidade de radiação ao longo do espectro.

A fim de simular a temperatura na superfície do painel, adicionou-se uma camada de vidro, com espessura de $4 \mathrm{~mm}$, na interface entre o bloco sólido e o ar.

A avaliação das perdas na eficiência do painel foram feitas com base nos dados de placa do painel usado como referência para modelagem e simulação. As características elétricas e térmicas do painel estão apresentadas na Tabela 1 .

\section{RESULTADOS}

Na Figura 3 são apresentados os resultados da simulação para a temperatura na interface dos domínios. As faixas de temperatura obtidas para cada simulação são representadas pelo gradiente de cores, variando do azul, representando a menor temperatura obtida, ao vermelho, correspondente ao maior valor desta variável. Para o caso em que $\mathrm{v}=0 \mathrm{~m} / \mathrm{s}$ (detalhe I), o programa indicou uma amplo espectro de valores de temperatura, sendo necessário fixar um limite. Como a temperatura máxima de operação na célula é $85^{\circ} \mathrm{C}(358 \mathrm{~K})$, escolheu-se esta temperatura como valor máximo. Nas demais simulações, é possível de se observar a variação no campo da temperatura no painel, devido a influência da circulação de ar. Para $\mathrm{v}=2,5 \mathrm{~m} / \mathrm{s}$ (detalhe II), por exemplo, foi obtida uma variação de temperatura de $17,5^{\circ} \mathrm{C}$ em relação a temperatura obtida para $\mathrm{v}=0$. Comparando-se os valores máximos das temperaturas indicados nas simulações com $\mathrm{v}=0 \mathrm{e} \mathrm{v}=7,5 \mathrm{~m} / \mathrm{s}$ (detalhe IV), observa-se uma variação de $48,75 \%$. Os valores máximos de temperatura obtidos nas simulações e os percentuais previstos de perda de potência estão apresentados na Tabela 2

Na Figura 4 é apresentada a variação do percentual de redução da potência em função da temperatura da célula. Da mesma forma que na Figura 3, percebe-se uma redução acentuada no percentual de redução da potência à medida que se resfria o painel. Também se observou uma amplitude cada vez menor entre valores sucessivos desta variável, o que indica que, quanto mais afastada a temperatura estiver do valor padrão de teste, maior será o percentual de melhora a se obter com o resfriamento do painel. Comparando-se os valores máximos e mínimos do percentual de perda de potência,

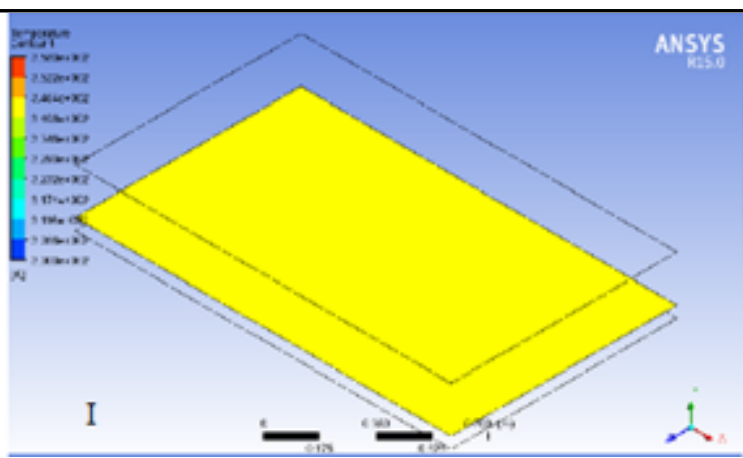

(a)

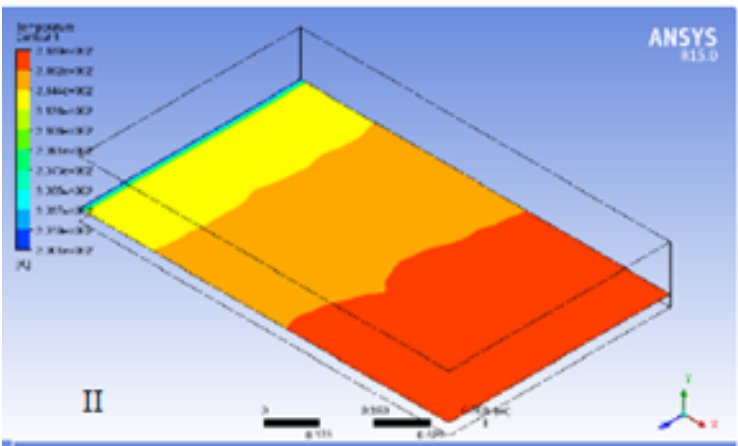

(b)

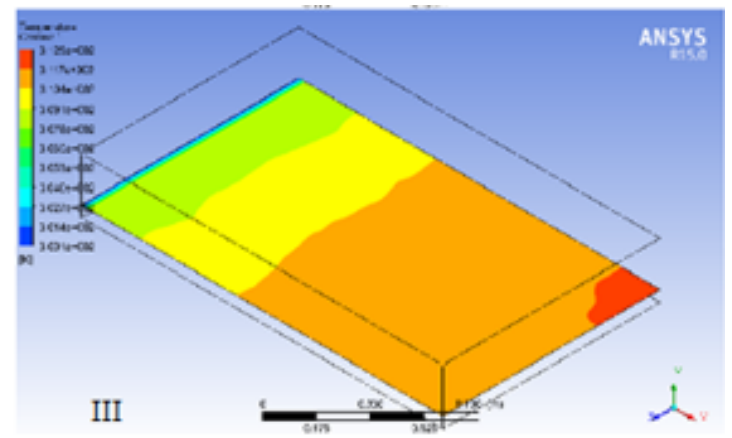

(c)

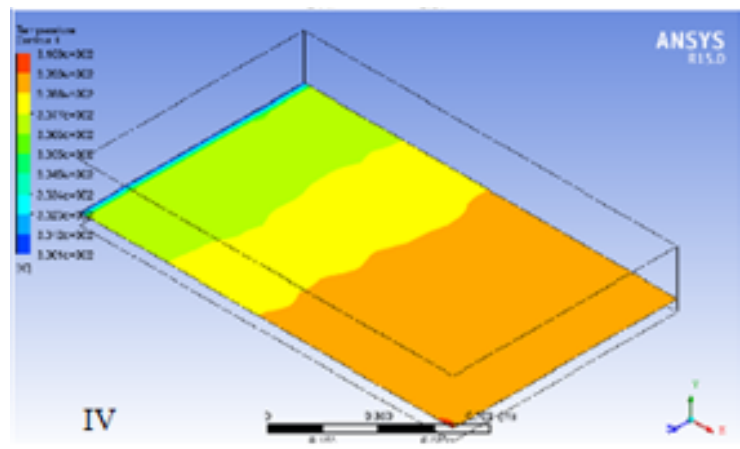

(d)

Figura 3: Temperatura na interface para as 4 condições a) $v=0 \mathrm{~m} / \mathrm{s}$ b) $v=2,5 \mathrm{~m} / \mathrm{s} \mathrm{c}$ ) $\mathrm{v}=5 \mathrm{~m} / \mathrm{s} \mathrm{d}) \mathrm{v}=7,5 \mathrm{~m} / \mathrm{s}$ 
Tab 1: Características térmicas do painel

\begin{tabular}{l|l}
\hline Eficiência do módulo (STC) & $15,40 \%$ \\
\hline Temperatura nominal da célula (NOCT) & $46+/-2{ }^{\circ} \mathrm{C}$ \\
\hline Coeficiente de temperatura para Pmáx & $-0,42 \% /{ }^{\circ} \mathrm{C}$ \\
\hline Gama de temperaturas operacionais & $-40{ }^{\circ} \mathrm{Ca} 85{ }^{\circ} \mathrm{C}$ \\
\hline Redução média da eficiência em caso de redução da intensidade para $200 \mathrm{~W} / \mathrm{m}^{2}$ & $1,90 \%$ \\
\hline
\end{tabular}

Tab 2: Quadro resumo dos resultados

\begin{tabular}{l|l|l|l} 
Simulação & Velocidade $(\mathrm{m} / \mathrm{s})$ & Temperatura máxima $\left({ }^{\circ} \mathrm{C}\right)$ & $\%$ queda \\
\hline 1 & 0,00 & 72,00 & 19,74 \\
\cline { 3 - 3 } 2 & 45,00 & 8,40 \\
3 & 2,50 & 40,00 & 6,30 \\
\hline 4 & 5,00 & 36,90 & 4,99 \\
\cline { 1 - 1 }$\%$ de variação na temperatura & 48,75
\end{tabular}

observa-se uma variação de $74,72 \%$.

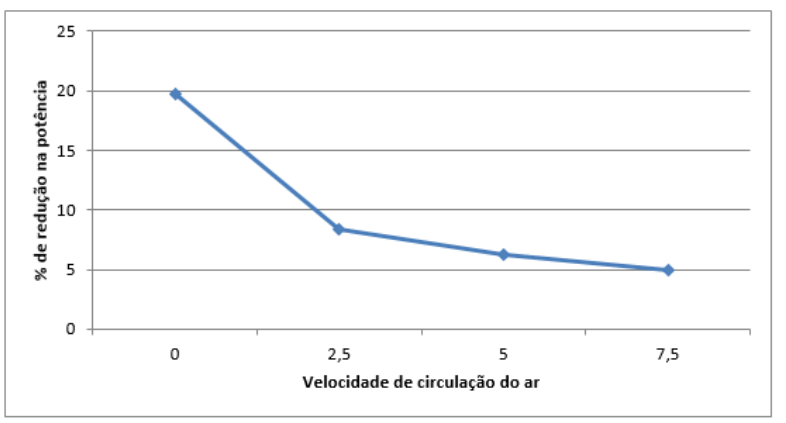

Figura 4: percentual de redução da potência em função da temperatura da célula

\section{CONCLUSÕES}

Neste artigo, abordou-se a influência da temperatura na potência de um painel solar, considerando-se apenas a incidência direta da radiação. Comparando-se os percentuais de perda de potência em painéis operando em condições de campo presentes na literatura com os calculados a partir dos resultados das simulações, observou-se que estes foram bastantes expressivos, ressaltando a necessidade de um sistema de resfriamento para se garantir um bom funcionamento dos painéis. A variação entre os percentuais de redução na potência nos casos v=0 e v=7,5 m/s foi de $74,72 \%$, enquanto que a variação na temperatura foi de $48,75 \%$.

Algumas dificuldades foram encontradas ao longo do estudo. $\mathrm{O}$ vidro utilizado na cobertura de painéis fotovoltaicos recebe um tratamento diferenciado, propriedade esta não presente no material existente na biblioteca do software, o que pode ter gerado uma fonte de erros na simulação. Além disso, como a estrutura do sólido foi considerada como um bloco feito de alumínio, sem considerar o design da superfície traseira do módulo, que auxilia nas trocas de calor, as temperaturas obtidas podem estar acima do que se observaria em condições de campo.

\section{REFERÊNCIAS}

BORGES NETO, M. R.; CARVALHO, P. C. M. Geração de Energia Elétrica Fundamentos. 1. ed. Fortaleza: Ed. Érica, 2012.

CARDONA, M. S.; LÓPEZ, M. L. Performance analysis of a grid-connected photovoltaic system Energy. New York: , 1999.

CRESESB/CEPEL. Energia Solar: Princípio e Aplicações. Rio de Janeiro: CRESESB - Centro de Referência para Energia Solar e Eólica Sérgio de Salvo Brito; CEPEL - Centro de pesquisas de Energia Elétrica. Sistema Eletrobrás, 2006.

GNOATTO, E.; DALLACORT, R.; RICIERI, R.; LIMA, S. de; FERRUZI, Y. Determinação da curva característica de um painel fotovoltaico em condições reais de trabalho. SIGCOMM Comput. Commun. Rev., Maringá-PR, p. 191-196, 2005.

GOKILAVANI, N.; PRABHAKARAN, D.; KANNADASAN, T. Experimental studies and CFD modeling on solar distillation system. International Journal of Innovative Research in Science, Engineering and Technology, v. 3, n. 9, p. 356-363, 2014.

GTES. Manual de Engenharia para Sistemas

Fotovoltaicos. Edição especial. Rio de Janeiro: Editora 
CEPEL/CRESESB, GTES - Grupo de Trabalho de Energia Solar, 2014.

GUENOUNOU, A.; MALEK, A.; AILLERIE, M. Comparative performance of $\{\mathrm{PV}\}$ panels of different technologies over one year of exposure: Application to a coastal mediterranean region of algeria. Energy Conversion and Management, v. 114, p. 356 - 363, 2016.

GXASHEKA, A. R.; DYK, E. E. van; MEYER, E. L. Evaluation of performance parameters of PV modules deployed outdoors. Renewable Energy, v. 30, n. 4, p. $611-620,2005$.

HERNANDEZ, R.; EASTER, S.; MURPHYMARISCAL, M.; MAESTRE, F.; TAVASSOLI, M.; ALLEN, E.; BARROWS, C.; BELNAP, J.; OCHOA-HUESO, R.; RAVI, S. et al. Environmental impacts of utility-scale solar energy. Renewable and Sustainable Energy Reviews, Elsevier, v. 29, p. 766-779, 2014.

MICHELS, J. S. R. Influência de fatores ambientais sobre o desempenho de um sistema de bombeamento fotovoltaico: um estudo de caso. Revista Agroambiental, v. 3, n. 3, p. 51-55, 2011.

MICHELS, R.; SANTOS, J. A.; KAWANAGH, E. G. E.; FISCHBORN, M.; HALMEMAN, M. Influência da temperatura sobre a potência de saída de um painel de silício policristalino na região oeste paranaense. In: CONGRESSO INTERNACIONAL SOBRE GERAÇÃO DISTRIBUIDA E ENERGIA NO MEIO RURAL. Fortaleza: , 2008. v. 7, n. 34. Disponível em: <http://nipeunicamp.org.br/agrener/ anais/2008/Artigos/51.pdf> Acesso em: 01 Junho 2016.

SACRAMENTO, E. M. do; CARVALHO, P. C. M.; ARAÚJO, J. C. de; RIFFEL, D. B.; CORRÊA, R. M. da C.; PINHEIRO NETO, J. S. Scenarios for use of floating photovoltaic plants in brazilian reservoirs. IET Renewable Power Generation, IET, v. 9, n. 8, p. 1019 1024, 2015.

SAHU, A.; YADAV, N.; SUDHAKAR, K. Floating photovoltaic power plant: A review. Renewable and Sustainable Energy Reviews, v. 66, p. 815 - 824, 2016.

TOLMASQUIM, M. Energia Renovável: hidráulica, biomassa, eólica, solar, oceânica. Rio de Janeiro: Empresa de Pesquisa Energética, 2016.

VILLALVA, M.; GAZOLI, J. Energia Solar Fotovoltaica: conceitos e aplicações. 1. ed. São Paulo: Ed. Erica, 2012.
YINGLI. Folha de Dados do Painel Solar Fotovoltaico Yingli YL260P $29 b$ (260 Wp). 2016. Disponível em: <wWw.energyshop. com.br/produto/placa-solar-yingli-260w/ painel-solar-fotovoltaico-yingli-260w>. Acesso em: 31 maio 2016. 